\title{
Identification of Prognostic Risk Factors for Transient and Persistent Lymphedema after Multimodal Treatment for Breast Cancer
}

\section{Myungsoo Kim, MD ${ }^{1,2}$ \\ Kyung Hwan Shin, MD, PhD ${ }^{1,3}$ \\ So-Youn Jung, MD \\ Seeyoun Lee, MD \\ Han-Sung Kang, MD \\ Eun Sook Lee, MD' \\ Seung Hyun Chung, MD \\ Yeon-Joo Kim, MD' \\ Tae Hyun Kim, MD \\ Kwan Ho Cho, MD}

${ }^{1}$ Research Institute and Hospital, National Cancer Center, Goyang, ${ }^{2}$ Department of Radiation Oncology, Incheon St. Mary's Hospital, College of Medicine,

The Catholic University of Korea, Incheon, ${ }^{3}$ Department of Radiation Oncology, Seoul National University

College of Medicine, Seoul, Korea

Correspondence: Kyung Hwan Shin, MD, PhD Department of Radiation Oncology, Seoul National University College of Medicine, 101 Daehak-ro, Jongno-gu, Seoul 03080, Korea Tel: 82- 2-2072-2524

Fax: 82-2-765-3317

E-mail: radiat@snu.ac.kr

Received December 1, 2015

Accepted January 19, 2016

Published Online February 3, 2016

\section{Purpose}

The purpose of this study is to identify risk factors for transient lymphedema (TLE) and persistent lymphedema (PLE) following treatment for breast cancer.

\section{Materials and Methods}

A total of 1,073 patients who underwent curative breast surgery were analyzed. TLE was defined as one episode of arm swelling that had resolved spontaneously by the next followup; arm swelling that persisted over two consecutive examinations was considered PLE.

\section{Results}

At a median follow-up period of 5.1 years, 370 cases of lymphedema were reported, including 120 TLE (11.2\%) and 250 PLE (23.3\%). Initial grade 1 swelling was observed in 351 patients, of which 120 were limited to TLE (34\%), while the other 231 progressed to PLE (66\%). All initial swelling observed in TLE patients was classified as grade 1. In multivariate analysis, chemotherapy with taxane and supraclavicular radiation therapy (SCRT) were associated with development of TLE, whereas SCRT, stage III cancer and chemotherapy with taxane were identified as risk factors for PLE $(p<0.05)$. The estimated incidence of TLE among initial grade 1 patients was calculated using up to three treatment-related risk factors (number of dissected axillary lymph nodes, SCRT, and taxane chemotherapy). The approximate ratios of TLE and PLE based on the number of risk factors were 7:1 (no factor), 1:1 (one factor), 1:2 (two factors), and 1:3 (three factors).

\section{Conclusion}

One-third of initial swelling events were transient, whereas the other two-thirds of patients experienced PLE. Estimation of TLE and PLE based on known treatment factors could facilitate prediction of this life-long complication.

\section{Introduction}

Lymphedema (LE) is a common and debilitating condition in breast cancer survivors characterized by regional swelling, typically in one or both arms [1]. It is characterized by an accumulation of protein-rich fluids caused by an interruption in the axillary lymphatic drainage from the arm [2]. Due to

\section{Key words}

Breast neoplasms, Transient lymphedema, Persistent lymphedema, Risk factors 
After the initial onset of symptoms, many patients experience a spontaneous resolution of swelling without progressing to a persistent condition. This transient swelling is typically of less than six-month duration [6].

At the time of initial swelling, determining whether a patient has either transient lymphedema (TLE) or persistent lymphedema (PLE) is important, particularly among PLE patients who must learn to cope with this life-long complication through the use of proactive education and management. In contrast, TLE patients, whose symptoms can be expected to resolve naturally, may benefit by avoiding unnecessary treatment. However, the incidence, time course of TLE, and risk factors associated with progression to PLE from initial swelling remain poorly understood. In this study, we analyzed the incidence and risk factors for development of TLE and PLE after initial swelling and estimated the ratio of TLE and PLE based on the presence of known treatment-related factors.

\section{Materials and Methods}

\section{Patients}

The study population consisted of 1,255 consecutive breast cancer patients who underwent curative breast surgery with axillary lymph node dissection (ALND) at the National Cancer Center, Korea between May 2004 and April 2009. Those with synchronous or metachronous contralateral breast cancer $(n=7)$ and those with a follow-up period of $<3$ years were excluded ( $\mathrm{n}=175)$. The remaining 1,073 patients were included in the current analysis, which was performed in accordance with the guidelines of the Institutional Review Board at the National Cancer Center of Korea.

\section{Treatment}

Surgery consisted of either modified radical mastectomy (MRM) or breast-conserving surgery (BCS), as necessary, based on the surgeon's recommendations and tumor characteristics. The general criteria for patient selection for BCS included a lack of initial extensive skin or chest wall involvement, absence of extensive microcalcifications or multifocal disease, the anticipated adequacy of residual breast tissue following BCS, and an absolute contraindication against breast irradiation. MRM was performed in all other patients. Standard ALND was performed with or without sentinel lymph node biopsy in all patients.

Adjuvant chemotherapy (ACT) consisted of six to eight courses of a non-taxane containing $(n=264)$ or taxane-con- taining $(\mathrm{n}=414)$ regimen. Neoadjuvant chemotherapy (NCT) consisted of four to six courses of anthracycline-based, taxane-based, or combined anthracycline-taxane therapy. Adjuvant hormonal suppression therapy using tamoxifen or an aromatase inhibitor was offered to 951 patients $(79 \%)$ with estrogen receptor-positive or progesterone receptor-positive tumors. Trastuzumab was administered for 1 year in patients with c-erbB2-overexpressing tumors.

Radiation therapy (RT) was delivered to all BCS and MRM patients with $\mathrm{pT} 3, \mathrm{pN} 2-\mathrm{pN} 3$, or $\mathrm{pN} 1$ with extracapsular invasion of the involved nodes for patients treated with ACT. All patients who received NCT were treated with RT, irrespective of the type of surgery. Planning computed tomography scans were obtained in all patients, and RT was performed using conventional techniques. Typically, 50.4 Gy were delivered in 28 fractions to the ipsilateral breast or chest wall [7]. After BCS, all patients received an electron boost (median, $10 \mathrm{~Gy}$ ) to the lumpectomy cavity. Ipsilateral axillary apex and supraclavicular RT (SCRT) was delivered to patients of $\mathrm{pN} 2$ to $\mathrm{pN} 3$ status who received ACT, and to all patients who received NCT (median, $45 \mathrm{~Gy}$ ). Daily posterior axillary boost was also delivered. Internal mammary nodal RT was administered to only 17 patients (5\%) with clinically positive internal mammary nodes. The initial planned dose of RT was completed in all cases.

\section{Measurement and assessment of LE}

Determination of LE was based on both objective (circumference measurement) and subjective (patient perception of arm edema) assessments, as described previously [3]. Beginning in 2004, measurement and assessment of LE was performed in all patients by a single physician (K.H.S.) as part of a prospective database. At each follow-up visit, patients were asked whether they were currently experiencing swelling, heaviness, thickness, tiredness, or pain in their affected arms, regardless of reported differences in arm size. Patients were also asked to recall preoperative arm condition, with no patient reporting preoperative arm swelling. Circumference measurements of the ipsilateral and contralateral arms at $10 \mathrm{~cm}$ above the antecubital fold were performed regularly every 6 months, beginning at least 6 months postoperatively until the last follow-up. Ipsilateral arm swelling of more than $5 \%$ of the circumferential difference without special conditions (such as a previous accident or injury to the contralateral arm) was considered objective LE. Graded scales combining subjective symptoms and the Common Terminology Criteria for Adverse Events ver. 4.0 were used for LE scoring. A difference of 5\%-10\% in arm measurement or only self-perception of arm swelling with less than 5\% measurement difference was scored as grade 1 ; arm measurement differences of $10 \%-30 \%$ or $>30 \%$ were scored as 
grades 2 or 3, respectively. Assessments were made beginning at least 6 months postoperatively and regularly every 6 months until the last follow-up.

LE events were defined as any case of arm swelling, including both objectively and subjectively defined incidents. TLE was defined as a single episode of arm swelling that resolved spontaneously at the next follow-up. PLE was defined as arm swelling that persisted over two consecutive follow-up examinations.

\section{Statistical analysis}

The rates of LE were calculated using the Kaplan-Meier method, and the development of LE was measured from the date of surgery. Univariate Cox proportional hazards models were used for evaluation of risk factors associated with the development of LE. The factors included age ( $<50$ years vs. $\geq 50$ years), body mass index (BMI; $<25 \mathrm{~kg} / \mathrm{m}^{2}$ vs. $\geq 25$ $\mathrm{kg} / \mathrm{m}^{2}$ ), T classification (T1-T2 vs. T3-T4), N classification (N0-N1 vs. N2-N3), stage (I-II vs. III), type of surgery (BCS vs. MRM), number of dissected axillary lymph nodes (N-ALN; $\leq 10$ vs. $>10$ ), and regimen of chemotherapy (not performed or chemotherapy without taxane vs. chemotherapy with taxane). Variables shown to be significant $(\mathrm{p}<0.05)$ in univariate analysis were selected for multivariate analysis. Differences between risk groups were estimated using the log-rank test. All statistical tests were two-sided, and statistical significance was defined as a $p$-value $<0.05$. Statistical analyses were performed using SPSS ver. 17.0 (SPSS Inc., Chicago, IL).

\section{Results}

A summary of patient and treatment characteristics is shown in Table 1 . The median age at diagnosis was 47 years (range, 26 to 81 years). In terms of disease stage, 213 patients (19.8\%) were diagnosed with stage I, 505 patients $(47.1 \%)$ with stage II, and 355 patients (33.1\%) with stage III. BCS was performed in 814 patients (75.9\%) and MRM in 259 patients (24.1\%). Median N-ALN was 11 (range, 5 to 41 ). ACT was administered to 678 patients (63.2\%) and NCT to 299 patients (27.9\%). Taxane-based regimen was administered to 706 patients $(65.8 \%)$ and non-taxane-based regimen to 271 patients $(25.3 \%)$. RT was administered to 965 patients $(89.9 \%)$; 410 patients (38.2\%) received breast or chest wall irradiation alone, and 555 patients (51.7\%) received breast irradiation with SCRT.
Table 1. Patient and treatment characteristics $(n=1,073)$

\begin{tabular}{|c|c|}
\hline Characteristic & No. $(\%)$ \\
\hline \multicolumn{2}{|l|}{ Age (yr) } \\
\hline Median (range) & $47(26-83)$ \\
\hline \multicolumn{2}{|l|}{ BMI $\left(\mathrm{kg} / \mathrm{m}^{2}\right)$} \\
\hline Median (range) & $23.6(17.1-42.5)$ \\
\hline \multicolumn{2}{|l|}{ Stage } \\
\hline I & $213(19.8)$ \\
\hline II & $505(47.1)$ \\
\hline III & 355 (33.1) \\
\hline \multicolumn{2}{|l|}{$\mathrm{T}^{\mathrm{a})}$} \\
\hline $\mathrm{T} 1$ & $435(40.5)$ \\
\hline $\mathrm{T} 2$ & $536(50.0)$ \\
\hline T3 & $74(6.9)$ \\
\hline $\mathrm{T} 4$ & $28(2.6)$ \\
\hline \multicolumn{2}{|l|}{$\mathrm{N}^{\mathrm{a})}$} \\
\hline N0 & $312(29.1)$ \\
\hline N1 & $433(40.3)$ \\
\hline N2 & $250(23.3)$ \\
\hline N3 & $78(7.3)$ \\
\hline \multicolumn{2}{|l|}{ Type of surgery } \\
\hline BCS & 814 (75.9) \\
\hline Mastectomy & $259(24.1)$ \\
\hline \multicolumn{2}{|l|}{ N-ALNs } \\
\hline$\leq 10$ & $489(45.6)$ \\
\hline $11-20$ & $470(43.8)$ \\
\hline$\geq 21$ & $114(10.6)$ \\
\hline \multicolumn{2}{|l|}{ Chemotherapy } \\
\hline Not done & $96(8.9)$ \\
\hline $\mathrm{ACT}$ & $678(63.2)$ \\
\hline NCT & $110(10.3)$ \\
\hline NCT and ACT & 189 (17.6) \\
\hline \multicolumn{2}{|l|}{ Radiation therapy } \\
\hline Not done & 108 (10.1) \\
\hline Breast only & $410(38.2)$ \\
\hline Breast with SCRT & $555(51.7)$ \\
\hline \multicolumn{2}{|l|}{ Hormonal therapy } \\
\hline No & $222(20.7)$ \\
\hline Yes & $851(79.3)$ \\
\hline
\end{tabular}

BMI, body mass index; BCS, breast-conserving surgery; $\mathrm{N}$-ALNs, number of dissected axillary lymph nodes; ACT, adjuvant chemotherapy; NCT, neoadjuvant chemotherapy; SCRT, supraclavicular radiation therapy. ${ }^{\text {a) }}$ Clinical stage in NCT.

\section{Incidence and time course of LE}

The median follow-up duration from the date of surgery was 5.1 years (range, 3.0 to 8.7 years). LE events were reported in 370 patients $(34.5 \%)$, consisting of 120 patients $(11.2 \%)$ with TLE and $250(23.3 \%)$ with PLE. The final LE 

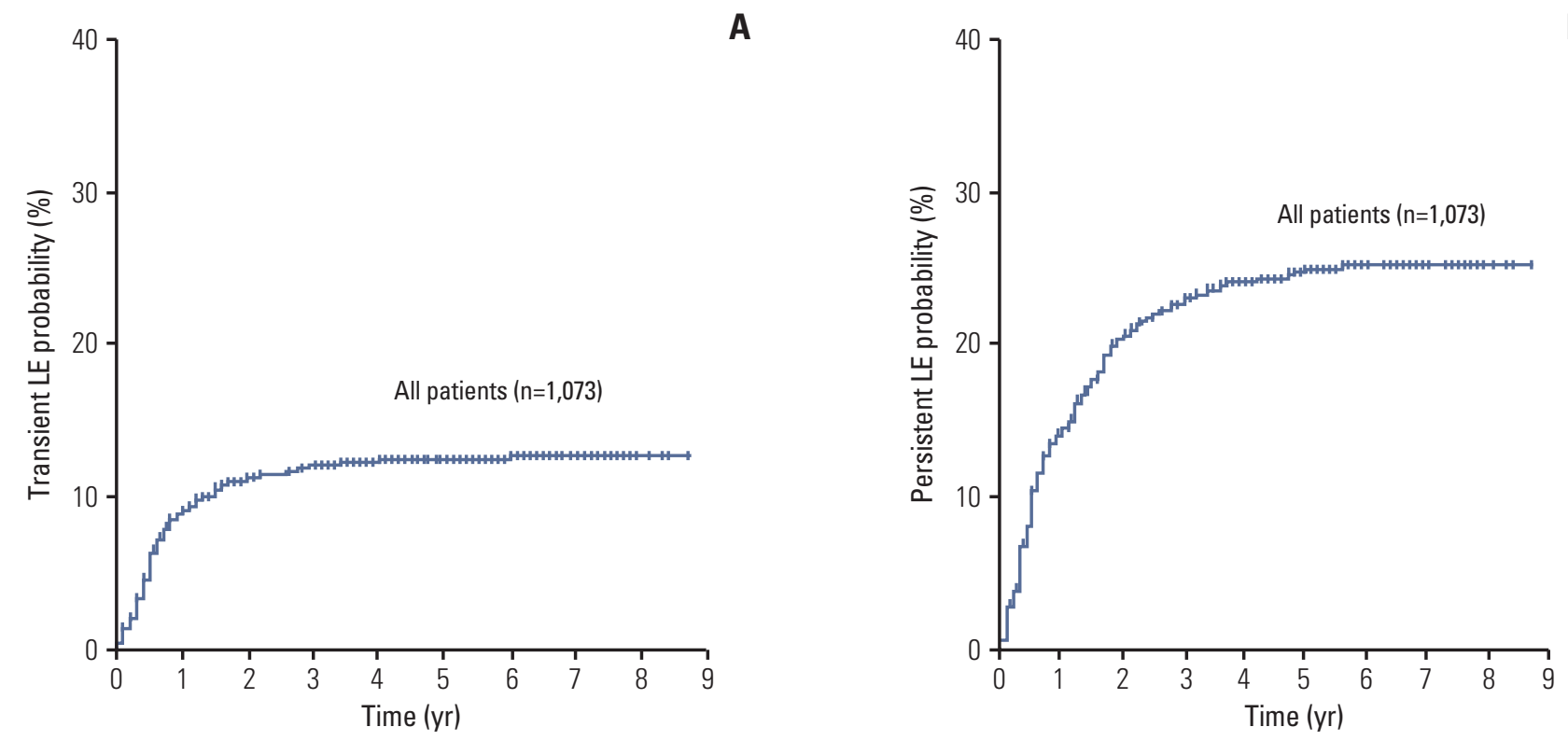

Fig. 1. Kaplan-Meier curves of the cumulative incidence of breast cancer-related lymphedema (LE) for transient (A) and persistent (B).

grade of 250 PLE patients was grade 1 in 188, grade 2 in 60 , and grade 3 in 2. Initial LE grade of all 370 patients was grade 1 in 351 (32.7\%) and grade 2 in 19 (1.8\%). A total of 120 of 351 patients who were initial grade $1 \mathrm{LE}$ were limited to TLE $(34 \%)$ and the remaining 231 patients progressed to PLE $(66 \%)$. The initial swelling for TLE patients was all grade 1 edema. Among the 119 grade 1 patients who had both subjective symptoms and objective differences at initial swelling, $95(80 \%)$ progressed to PLE. All 19 patients with grade 2 LE at the time of initial diagnosis progressed to PLE. Among PLE patients, 49 of 231 patients initially diagnosed as grade 1 eventually progressed to grade 2 , with one patient progressing to grade 3 .

The overall 5-year cumulative incidence of TLE and PLE was $12.5 \%$ and $25.3 \%$, respectively (Fig. 1). Among TLE patients, LE events occurred within 1 year after surgery in $73 \%$ of cases, 2 years in $91 \%$ of cases, and 3 years in $98 \%$ of cases. In PLE patients, LE events occurred within 1 year after surgery in $58 \%$ of cases, 2 years in $83 \%$ of cases, and 3 years in $93 \%$ of cases.

\section{Risk factors correlated with LE}

Results of univariate analysis of patient-, disease-, and treatment-related factors associated with development of TLE and PLE are shown in Table 2. In univariate analysis, higher rates of TLE development showed significant correlation with advanced stage at diagnosis (III), N-ALN (> 10), chemotherapy regimen (with taxane), and RT (breast with SCRT); higher rates of PLE development showed correlation with advanced stage at diagnosis (III), type of surgery (MRM), N-ALN (>10), chemotherapy regimen (with taxane), and RT (breast with SCRT). Patients with more advanced stages of disease showed higher rates of LE (12.4\% in stage III vs. $10.6 \%$ in stage I or II for TLE, $41.7 \%$ vs. $14.2 \%$ for PLE; $\mathrm{p}<0.001)$. The rates of PLE in patients who underwent BCS and MRM were $21.6 \%$ and $28.2 \%$, respectively $(p=0.02)$. The LE rates for N-ALNs $\leq 10$ and $>10$ for TLE were $9 \%$ and $13 \%$, respectively $(\mathrm{p}=0.001)$, compared with $13.7 \%$ and $31.3 \%$, respectively, for PLE $(\mathrm{p}<0.001)$. Regarding chemotherapy regimens, chemotherapy with taxane showed significant association with an increased risk of both TLE and PLE, compared with patients receiving no chemotherapy or chemotherapy without taxane $(13.5 \%$ vs. $6.8 \%$ for TLE, $33.0 \%$ vs. $4.6 \%$ for PLE; $\mathrm{p}<0.001)$. Incidences of TLE and PLE were significantly higher in patients who received breast RT with SCRT than in patients who received no RT or breast RT only (14.2\% vs. $7.9 \%$ for TLE, $37.8 \%$ vs. $7.7 \%$ for PLE; $\mathrm{p}<0.001$ ). Factors not significantly associated with LE outcomes included age and BMI in both TLE and PLE, with TLE also showing no association with type of surgery.

In multivariate analysis using a stepwise backward selection procedure, chemotherapy with taxane (hazard ratio [HR], 1.86; $\mathrm{p}=0.03$ ) and breast RT with SCRT (HR, 2.03; $\mathrm{p}=0.003$ ) were independent risk factors for TLE development (Table 3 ). The advanced stage and N-ALN did not affect TLE. 
Table 2. Univariate analysis of risk factors associated with breast cancer-related lymphedema

\begin{tabular}{|c|c|c|c|c|c|}
\hline Classification & Total $(n=1,073)$ & Transient LE $(\%)(n=120)$ & p-value & Persistent LE $(\%)(\mathrm{n}=\mathbf{2 5 0})$ & p-value \\
\hline \multicolumn{6}{|l|}{ Age (yr) } \\
\hline$<50$ & 690 & $80(11.6)$ & 0.86 & $149(21.6)$ & 0.06 \\
\hline$\geq 50$ & 383 & $40(10.4)$ & & $101(26.4)$ & \\
\hline \multicolumn{6}{|l|}{ BMI $\left(\mathrm{kg} / \mathrm{m}^{2}\right)^{\mathrm{a})}$} \\
\hline$<25$ & 713 & $77(10.8)$ & 0.40 & $161(22.6)$ & 0.47 \\
\hline$\geq 25$ & 351 & $43(12.3)$ & & $84(23.9)$ & \\
\hline \multicolumn{6}{|l|}{ Stage } \\
\hline I or II & 718 & $76(10.6)$ & 0.001 & $102(14.2)$ & $<0.001$ \\
\hline III & 355 & $44(12.4)$ & & $148(41.7)$ & \\
\hline \multicolumn{6}{|l|}{$\mathrm{T}$} \\
\hline $\mathrm{T} 1-\mathrm{T} 2$ & 971 & $107(11.0)$ & 0.10 & $209(21.5)$ & $<0.001$ \\
\hline $\mathrm{T} 2-\mathrm{T} 4$ & 102 & $13(12.7)$ & & $41(40.2)$ & \\
\hline \multicolumn{6}{|l|}{$\mathbf{N}$} \\
\hline N0-N1 & 745 & $79(10.6)$ & 0.001 & $112(15.0)$ & $<0.001$ \\
\hline N2-N3 & 328 & $41(12.5)$ & & $138(42.1)$ & \\
\hline \multicolumn{6}{|l|}{ Type of surgery } \\
\hline BCS & 814 & $94(11.6)$ & 0.88 & $177(21.7)$ & 0.02 \\
\hline Mastectomy & 259 & $26(10.0)$ & & $73(28.2)$ & \\
\hline \multicolumn{6}{|l|}{ N-ALNs } \\
\hline$\leq 10$ & 489 & $44(9.0)$ & 0.001 & $67(13.7)$ & $<0.001$ \\
\hline$>10$ & 584 & $76(13.0)$ & & $183(31.3)$ & \\
\hline \multicolumn{6}{|l|}{ Regimen of chemotherapy } \\
\hline Not done or without taxane & 367 & $25(6.8)$ & $<0.001$ & $17(4.6)$ & $<0.001$ \\
\hline Chemotherapy with taxane & 706 & $95(13.5)$ & & $233(33.0)$ & \\
\hline \multicolumn{6}{|l|}{ Radiation therapy } \\
\hline Not done or breast only & 518 & $41(7.9)$ & $<0.001$ & $40(7.7)$ & $<0.001$ \\
\hline Breast with SCRT & 555 & $79(14.2)$ & & $210(37.8)$ & \\
\hline
\end{tabular}

LE, lymphedema; BMI, body mass index; BCS, breast-conserving surgery; N-ALNs, number of dissected axillary lymph nodes; SCRT, supraclavicular radiation therapy. ${ }^{\text {a) }}$ Analysis except nine patients without BMI information.

Table 3. Multivariate analysis of risk factors associated with breast cancer-related lymphedema

\begin{tabular}{|c|c|c|c|c|}
\hline \multirow{2}{*}{ Variable } & \multicolumn{2}{|c|}{ Transient LE } & \multicolumn{2}{|c|}{ Persistent LE } \\
\hline & HR $(95 \% \mathrm{CI})$ & p-value & HR $(95 \%$ CI) & p-value \\
\hline Stage (III) & $0.94(0.61-1.44)$ & 0.78 & $1.49(1.12-1.97)$ & 0.006 \\
\hline N-ALNs (>10) & $1.18(0.78-1.77)$ & 0.43 & $1.21(0.91-1.63)$ & 0.20 \\
\hline Regimen of chemotherapy with taxane & $1.86(1.07-3.24)$ & 0.03 & $3.74(2.11-6.63)$ & $<0.001$ \\
\hline Radiation therapy (breast with SCRT) & $2.03(1.26-3.26)$ & 0.003 & $2.74(1.80-4.17)$ & $<0.001$ \\
\hline
\end{tabular}

LE, lymphedema; HR, hazard ratio; CI, confidence interval; N-ALNs, number of dissected axillary lymph nodes; SCRT, supraclavicular radiation therapy.

For PLE development, advanced stage (HR, 1.49; $\mathrm{p}=0.006)$, chemotherapy with taxane (HR, 3.74; $\mathrm{p}<0.001)$, and breast RT with SCRT $(\mathrm{HR}, 2.74 ; \mathrm{p}<0.001)$ were independent risk factors.

\section{Estimation of TLE and PLE by treatment factors}

The treatment-related factors N-ALN, SCRT, and taxane chemotherapy have been shown to significantly affect the development of LE $[3,8,9]$; therefore we examined whether 
Table 4. Incidence of transient and persistent lymphedema in patients with initial grade 1 swellings according to the number of risk factors

\begin{tabular}{lcccc} 
No. of risk factors ${ }^{a}$ & No. of patients $(\mathrm{n}=351)$ & Transient lymphedema & Persistent lymphedema & Approximate ratio \\
\hline 0 & 15 & $13(87)$ & $2(13)$ & $7: 1$ \\
1 & 36 & $16(44)$ & $20(56)$ & $1: 1$ \\
2 & 107 & $39(36)$ & $68(64)$ & $1: 2$ \\
3 & 193 & $52(27)$ & $141(73)$ & $1: 3$ \\
\hline
\end{tabular}

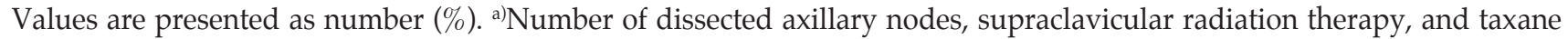
chemotherapy.

the tendency of TLE and PLE could be estimated using those factors. The incidences of TLE and PLE relative to the number of treatment factors in 351 patients with initial grade 1 swelling are shown in Table 4 . Among 15 patients who had no risk factors, 13 patients $(87 \%)$ were limited to TLE and two $(13 \%)$ were defined as PLE. Sixteen of 36 patients $(44 \%)$ with one risk factor were TLE; the remaining $20(56 \%)$ were PLE. The approximate ratio in these scenarios was 1:1. The incidences of TLE and PLE in 107 patients with two risk factors increased to $36 \%(n=39)$ and $64 \%(n=68)$, respectively, to an approximate ratio of 1:2. Of the 193 patients with three risk factors, the approximate ratio of TLE to PLE was 1:3.

\section{Discussion}

LE is an adverse and chronic condition affecting a considerable number of breast cancer survivors. The incidence of LE among patients with breast cancer varies from less than $5 \%$ with lumpectomy alone to more than $60 \%$ when treatment includes mastectomy with ALND and RT [10]. Reports of the incidence of each condition are also inconsistent due to the variation in the criteria used to define LE and the variety of assessment techniques [11]. Here, we used a grading scale combining both the objective method of circumference measurement and the subjective method of patient perception of arm edema to complement the accuracy of each method [12]. LE was then further subdivided in TLE and PLE according to presence or absence of arm swelling during the 6-month follow-up examination. PLE (chronic) is of greater than 6 months in duration, whereas TLE typically lasts less than 6 months and is related to pitting edema without brawny skin changes [6]; this distinction allowed for a clear demarcation between the two types of LE.

Examination of TLE is important, as the natural history of the condition, including incidence, time course, and risk factors, are not well documented in the literature. Werner et al. [13] reported that transient and persistent edema occurred in $7 \%$ and $12 \%$ of women, respectively, in a study of 282 patients with stage I or II breast cancer who received BCS with ALND. Higher levels were reported in a study by Norman et al. [14] in which $23.1 \%$ of patients experienced mild LE that generally resolved, or diminished to the point of only mild flare-ups. In terms of timing, the majority of swelling in the first year was shown to be largely transient, with factors including exercise and taxane chemotherapy associated with swelling incidence [4]. In our current study, the overall 5 -year cumulative incidence of TLE was $12.5 \%$, while that of PLE was $25.3 \%$. The onset of first swelling in patients with TLE was generally earlier compared to those with PLE, possibly due to acute swelling associated with three breast cancer treatment modalities. Of the TLE patients, 73\% developed swelling within 1 year after surgery, compared to only $58 \%$ of PLE patients.

A recent meta-analysis identified chemotherapy as a risk factor for LE development [1]. We have since expanded on this finding, showing a clear association between chemotherapy and an increased risk of LE in the context of both ACT and NCT [3,9], with taxane identified as an independent risk factor for LE [8]. In the current study, chemotherapy with taxane, compared with no chemotherapy or chemotherapy without taxane, increased the risk of TLE and PLE. This observation was unsurprising, as taxane is known to cause generalized edema by increasing the amount of extracellular fluid in the interstitial compartment $[4,8,15]$. The increased incidence of LE in the ipsilateral arm following taxane-based chemotherapy has been shown to persist for at least 6 months after cessation chemotherapy; however, generalized swelling in the legs and contralateral arm resolved within 6 months after chemotherapy [15]. Similarly, Kilbreath et al. [4] reported that taxane-based chemotherapy causes preferential transient swelling in the first year following surgery.

ALND and SCRT are considered the most important risk factors for LE [1]. The risk of LE has been shown to increase in response to an increase in N-ALNs [16,17]. Here, N-ALN $>10$ was identified as a risk factor for both PLE and TLE by univariate analysis. SCRT was also a strong contributing factor, associated with a 2- to 4.5 -fold increase in the risk of LE 
[3]. Similarly, Coen et al. [18] reported a significant increase in the risk of LE from $1.8 \%$ to $8.9 \%$ over a 10-year follow-up period in patients with BCS following the addition of SCRT. In this study, SCRT was identified as an independent risk factor for development of not only PLE but also TLE in a multivariate analysis.

Because LE is progressive, and early diagnosis could lead to more effective treatment, diagnosis of LE at the earliest possible stage is important. At first presentation of arm swelling, understanding of risk factors associated with PLE and predicting whether this condition is expected to be selflimiting or persistent may enable clinicians to mitigate PLE incidence and severity by providing proactive education and management. This study showed that the tendency of TLE and PLE after initial grade 1 swelling could be estimated using a combination of three treatment-related risk factors: $\mathrm{N}-\mathrm{ALN}, \mathrm{SCRT}$, and taxane chemotherapy. In patients with no risk factor, $\sim 90 \%$ of swelling events were limited in scope, and had completely disappeared within 6 months. However, in all other patients, the likelihood of edema persisting or progressing to a more serious form increased according to the number of risk factors. The approximate ratio of TLE vs. PLE was 1:1 in patients with one risk factor, 1:2 in patients with two factors, and 1:3 in those with three risk factors. As mentioned above, 95 of 119 grade 1 patients $(80 \%)$ who had both subjective symptoms and an objective arm circumference difference progressed to PLE, implying that patients who had at least one risk factor or both subjective and objective symptoms of LE may require more careful follow-up. Patient-related factors-such as obesity, hand use, injury, and infection - could be modified to reduce the risk for these patients [1]. In a study of 109 patients with mild LE at LE diagnosis by Bar Ad et al. [19], 52 (48\%) progressed to more severe stages at some point during the first 5 years of followup. In our study, 49 of 351 patients (14\%) initially diagnosed as grade 1 eventually progressed to grade 2 , with one patient progressing to grade 3 .

Limitations of our study include the lack of preoperative baseline information on arm circumference or the ability to interview patients about arm swelling. In a meta-analysis by DiSipio et al. [1], even in the absence of pretreatment LE status, prevalence was thought to be a reasonable estimate of incidence because the proportion of female patients with LE before surgery for breast cancer has been reported to be very low. Another limitation is the accuracy of the subjective and objective definitions for LE. The subjective patient's perception of arm edema was dependent on patient recall or swelling sense at the time of the outpatient clinic visit. For objective measurements, only the upper arm circumference was measured; hence, localized forearm or hand edema would not have been detected, although subjective assessments may have been sufficient to overcome this limitation.
In addition, we used a percentage difference rather than an actual difference in arm circumference for consideration of different BMI among patients and body weight changes overtime in each patient $[1,12]$. The other limitation is that our study did not include data regarding treatment of LE. However, in our hospital, education on the modification of patient-related factors such as reducing excessive arm use, avoiding arm infection of injury, and weight control was mandatorily provided to all breast cancer patients regardless of swelling episodes. In addition, most patients with objective LE at the first swelling episodes were managed using a compression stocking or bandage.

\section{Conclusion}

In conclusion, one-third of initial swelling events were transient in nature, with the remaining two-thirds progressing to a more persistent form of LE. Among patients with TLE, chemotherapy with taxane and RT covering the breast and supraclavicular region were identified as independent risk factors. The approximate incidence ratios of TLE and PLE in patients with initial grade 1 edema were estimated according to treatment-related factors, which could facilitate prediction and treatment of this life-long, undesired complication.

\section{Conflicts of Interest}

Conflict of interest relevant to this article was not reported.

\section{Acknowledgments}

This work was supported by Research Resettlement Fund for the new faculty of Seoul National University. 


\section{References}

1. DiSipio T, Rye S, Newman B, Hayes S. Incidence of unilateral arm lymphoedema after breast cancer: a systematic review and meta-analysis. Lancet Oncol. 2013;14:500-15.

2. Cemal Y, Pusic A, Mehrara BJ. Preventative measures for lymphedema: separating fact from fiction. J Am Coll Surg. 2011; 213:543-51.

3. Kim M, Kim SW, Lee SU, Lee NK, Jung SY, Kim TH, et al. A model to estimate the risk of breast cancer-related lymphedema: combinations of treatment-related factors of the number of dissected axillary nodes, adjuvant chemotherapy, and radiation therapy. Int J Radiat Oncol Biol Phys. 2013; 86:498-503.

4. Kilbreath SL, Lee MJ, Refshauge KM, Beith JM, Ward LC, Simpson JM, et al. Transient swelling versus lymphoedema in the first year following surgery for breast cancer. Support Care Cancer. 2013;21:2207-15.

5. Tsai RJ, Dennis LK, Lynch CF, Snetselaar LG, Zamba GK, Scott-Conner C. The risk of developing arm lymphedema among breast cancer survivors: a meta-analysis of treatment factors. Ann Surg Oncol. 2009;16:1959-72.

6. Brennan MJ. Lymphedema following the surgical treatment of breast cancer: a review of pathophysiology and treatment. J Pain Symptom Manage. 1992;7:110-6.

7. Park SH, Kim JC, Lee JE, Park IK. Virtual lymph node analysis to evaluate axillary lymph node coverage provided by tangential breast irradiation. Radiat Oncol J. 2015;33:50-6.

8. Jung SY, Shin KH, Kim M, Chung SH, Lee S, Kang HS, et al. Treatment factors affecting breast cancer-related lymphedema after systemic chemotherapy and radiotherapy in stage II/III breast cancer patients. Breast Cancer Res Treat. 2014;148:91-8.

9. Kim M, Park IH, Lee KS, Ro J, Jung SY, Lee S, et al. Breast cancer-related lymphedema after neoadjuvant chemotherapy. Cancer Res Treat. 2015;47:416-23.

10. Gartner R, Jensen MB, Kronborg L, Ewertz M, Kehlet H, Kroman N. Self-reported arm-lymphedema and functional impairment after breast cancer treatment: a nationwide study of prevalence and associated factors. Breast. 2010;19:506-15.
11. Harris SR, Hugi MR, Olivotto IA, Levine M; Steering Committee for Clinical Practice Guidelines for the Care and Treatment of Breast Cancer. Clinical practice guidelines for the care and treatment of breast cancer: 11. Lymphedema. CMAJ. 2001; 164:191-9.

12. McLaughlin SA, Wright MJ, Morris KT, Giron GL, Sampson MR, Brockway JP, et al. Prevalence of lymphedema in women with breast cancer 5 years after sentinel lymph node biopsy or axillary dissection: objective measurements. J Clin Oncol. 2008;26:5213-9.

13. Werner RS, McCormick B, Petrek J, Cox L, Cirrincione C, Gray $\mathrm{JR}$, et al. Arm edema in conservatively managed breast cancer: obesity is a major predictive factor. Radiology. 1991;180: $177-84$.

14. Norman SA, Localio AR, Potashnik SL, Simoes Torpey HA, Kallan MJ, Weber AL, et al. Lymphedema in breast cancer survivors: incidence, degree, time course, treatment, and symptoms. J Clin Oncol. 2009;27:390-7.

15. Lee MJ, Beith J, Ward L, Kilbreath S. Lymphedema following taxane-based chemotherapy in women with early breast cancer. Lymphat Res Biol. 2014;12:282-8.

16. Dominick SA, Madlensky L, Natarajan L, Pierce JP. Risk factors associated with breast cancer-related lymphedema in the WHEL Study. J Cancer Surviv. 2013;7:115-23.

17. Ahmed RL, Schmitz KH, Prizment AE, Folsom AR. Risk factors for lymphedema in breast cancer survivors, the Iowa Women's Health Study. Breast Cancer Res Treat. 2011;130: 981-91.

18. Coen JJ, Taghian AG, Kachnic LA, Assaad SI, Powell SN. Risk of lymphedema after regional nodal irradiation with breast conservation therapy. Int J Radiat Oncol Biol Phys. 2003;55: 1209-15.

19. Bar Ad V, Cheville A, Solin LJ, Dutta P, Both S, Harris EE. Time course of mild arm lymphedema after breast conservation treatment for early-stage breast cancer. Int J Radiat Oncol Biol Phys. 2010;76:85-90. 